\title{
Numerical Simulations of the Precessing Jets of SS433
}

\author{
E. Müller and W. Brinkmann \\ MPI für Astrophysik, Karl-Schwarzschild-Strasse 1, 85740 Garching, \\ Germany
}

\begin{abstract}
The unique Galactic object SS433 contains the best-studied relativistic jets, and their physical parameters (velocity, temperature, density) are known to an astronomically unprecedented accuracy, i.e., to factors of order unity. The comparison of numerical simulations of these jets with observations provides deeper insight into the SS433/W50 system and is ideal for testing our understanding of the jet phenomenon in general.
\end{abstract}

\section{Introduction}

The Galactic binary system SS433 emits two oppositely directed relativistic jets with velocities of $v_{j}=0.26 c$ which precess with a period of $P_{p r} \sim 163$ days and a precession angle of $\Theta=20^{\circ}$. The object has been studied for more than twenty years over the whole accessible energy range, from the radio to the $\mathrm{X}$-ray band, and thus the jets' physical parameters are known to an astronomically unprecedented accuracy (Margon 1984; Brinkmann et al. 1991).

However, several of the unique properties of the system remain unexplained: its evolutionary status, the masses of the two stars of the binary, the physical processes leading to the acceleration and the precession of the jets, and their interaction with the surrounding supernova remnant W50 where the enormous kinetic energy of the jets is deposited. Further, most of the parameters are not measured directly but are deduced from comparisons of observational data with "simple" hydrodynamic jet models, particularly in the X-ray band.

Recent X-ray observations with ROSAT (Brinkmann, Aschenbach, \& Kawai 1996) and ASCA (Yamauchi, Kawai, \& Aoki 1994) have enabled an analysis of the "terminal fate" of the jets interacting with the surrounding interstellar medium on spatial scales $>10^{19} \mathrm{~cm}$. Two experimental findings are of particular interest for the understanding of the physical processes responsible for the energy transfer: the angular region over which this transfer seems to occur is far less than the $\sim 40^{\circ}$ opening angle of the precession cone, and secondly, the X-ray spectrum appears to be featureless and thus, perhaps, of nonthermal origin.

\section{Numerical Calculations}

To understand the evolution and the braking of the jets by the interstellar medium we performed 3-dimensional, time dependent hydro-dynamical simulations of a precessing jet with parameters as deduced from the optical and 
X-ray observations. We used an Eulerian PPM code with $500 \times 120 \times 120$ zones, starting at a radial distance of $10^{15} \mathrm{~cm}$ from the jet's origin, with a non-uniform grid spacing. The angular region of the spherical grid was $120^{\circ}$ in both angular variables. At the inner (radial) boundary, the precessing jet was flowing in; otherwise, outflow boundary conditions were used. So far, the temporal evolution over about eight precessional periods of the jet has been calculated.

The propagating jet shows a large variety of highly complex structures and instabilities which are of great theoretical interest and which deserve further specific studies. Details of the calculations can be found in Müller \& Brinkmann (2000) — only some astrophysically relevant remarks shall be made here:

- The speed of the head of the jet is considerably less than the initial outflow velocity of $0.26 c$, due to the snow plough effect.

- In a complex flow pattern, matter from the propagating precessing jet is dispersed laterally outwards as well as transported inwards, towards the symmetry axis. Here, the matter piles up, reaching densities far higher than in the original conical outflow. This "secondary" jet might then form the observed narrow-cone X-ray structure.

- A calculation of the X-ray emission from the jet, by determining for each computational cell the equilibrium ionization structure (assuming cosmic abundances), and with that the thermal emission of the gas, shows that the emerging radiation has a nearly featureless spectrum. Only future $\mathrm{X}$-ray missions with highly improved spectral resolution will be able to discriminate this spectral form from a genuine power law.

\section{Summary}

SS433 provides an ideal diagnostic tool for our understanding of the jet phenomenon, and thus it is a prime target of current X-ray missions like AXAF, ASTRO-E, and XMM. Of particular interest on the theoretical side are largescale hydrodynamic simulations which can shed light onto the question of the terminal braking of jets and onto the modes of energy transfer of such jets to their surroundings.

\section{References}

Brinkmann, W., Aschenbach, B., \& Kawai, N. 1996, A\&A, 312, 306

Brinkmann, W., Kawai, N., Matsuoka, M., \& Fink, H. H. 1991, A\&A, 241, 112

Margon, B. 1984, ARA\&A, 22, 507

Müller, E., \& Brinkmann, W. 2000, A\&A, submitted

Yamauchi, S., Kawai, N., \& Aoki, T. 1994, PASJ, 46, L109 